\title{
THE USE OF PHYTOBENTHOS FOR THE ECOLOGICAL STATUS ASSESSMENT IN UPPER SAKARYA BASIN, TURKEY
}

\author{
CETIN, T. ${ }^{*}-$ DEMIR, N. ${ }^{2}$ \\ ${ }^{1}$ General Directorate of Water Management, Ministry of Agriculture and Forestry, Republic of \\ Turkey, 06560 Ankara, Turkey \\ ${ }^{2}$ Department of Fisheries and Aquaculture Engineering, Faculty of Agriculture, Ankara \\ University, 06110 Ankara, Turkey \\ (phone: + 90-312-596-1643; fax: +90-312-318-5298) \\ *Corresponding author \\ e-mail: tolga.cetin@tarimorman.gov.tr; phone: +90-312-207-5818; fax: +90-312-207-5573 \\ (Received 13 ${ }^{\text {th }}$ Apr 2019; accepted $1^{\text {st }}$ Jul 2019)
}

\begin{abstract}
In this study, it was aimed to use diatoms as representatives of phytobenthos for estimating the ecological status in Upper Sakarya Basin. Samples were taken seasonally from ten stations from April 2015 to December 2015. Stations 1, 2, 3, 4, 5, 6 and 7 were selected from rivers, stations 8 and 9 were selected from ponds which form Sakarya Springs. Station 10 was located at the outlet of Cifteler Aquaculture and Research Station. Diatoms were identified, counted and 19 diatom indices were calculated. Moreover, water temperature, dissolved oxygen, $\mathrm{pH}$, electrical conductivity were measured in $s i t u$, and total nitrogen, orthophosphate and alkalinity were analysed. Finally, ecological status was estimated by evaluating biological, chemical and hydromorphological status according to Water Framework Directive. In total, 96 diatom species were identified from samples and Achnanthidium minutissimum, Cymbella excisa, Craticula subminuscula and Fragilaria brevistriata were the dominant species. Among diatom indices, IDP index which shows a high correlation with total nitrogen was used for the evaluation of the biological status. As a result, ecological status of the station 2 was estimated as high (class I), the status of stations 1, 4, 7, 8, 9 and 10 was estimated as good (class II), and the status of stations 3, 5, 6 was estimated as moderate (class III).
\end{abstract}

Keywords: diatom, index, biological monitoring, reference site, water quality

\section{Introduction}

Biological, chemical and hydromorphological monitoring has been carried out in Turkey since 2011 within the scope of alignment of Water Framework Directive (WFD). Rivers, lakes, coastal and transitional waters were monitored in 25 river basins. Water bodies were delineated, typology system was established, and sensitive areas were determined for that purpose. Preparation of river basin management plans and identification of reference sites are still ongoing.

The WFD requires to assess the status of rivers and lakes by using numerous parameters, one of which is phytobenthos. "Macrophytes and phytobenthos" are treated as two separate biological quality elements, and diatoms are frequently considered as representatives of phytobenthos (Kelly, 2013). Ecological status assessment is based on the status of biological, hydromorphological and physicochemical quality elements, by comparing data with the reference conditions (Muxika et al., 2007).

Phytobenthos is composed of many groups of living organisms such as macroalgae. However, reliable methods are developed mostly for diatoms. Some countries such as Czech Republic, Austria, Germany and Spain developed assessment methods for nondiatom communities. For instance, the activities regarding the identification of 
macroalgae and development of indices are still ongoing. However, mostly diatoms are used for ecological status classification within the scope of the WFD.

Several studies were conducted on diatom indices for water quality assessment (Cemagref, 1982; Kelly and Whitton, 1995; Lenoir and Coste, 1996; Rott et al., 1999; Gomez and Licursi, 2001). Some studies were carried out in order to classify the water quality by using diatom indices in Turkey (Kalyoncu et al., 2009; Kivrak et al., 2012; Morkoyunlu Yüce and Gönülol, 2016; Atıc1 et al., 2018). In addition, other studies are also available which evaluate the phytobenthos for each river basin (Demir et al., 2017; Toudjani et al., 2017).

In diatom studies carried out in the Sakarya Basin, 117 epilithic diatoms were identified as a result of sampling from June 2006 to February 2007 from five stations in Felent Creek. Water quality was then assessed according to the Watanabe index (Watanabe et al., 1990) and the Sladecek index (Sladecek, 1986). In the light of results, organic pollution was recorded in summer, and species richness was higher mostly in winter than in summer (Solak et al., 2012). In total, 45 epipelic diatoms were identified in spring 2010 in Gürleyik stream, and the water quality was determined according to the Trophic Diatom Index (TDI) (Kelly and Whitton, 1995) and Biological Diatom Index (IBD) (Coste et al., 2009) as mesotrophic (Tokatl1, 2012).

The Upper Sakarya Basin which is covered by one of the largest river basins in Turkey (Sakarya Basin) was selected in this study. The aim of the study is to investigate the seasonal variation of diatom composition, and to estimate the ecological status with hydromorphological and physicochemical quality elements in the Upper Sakarya Basin.

\section{Materials and methods}

The Upper Sakarya Basin is located between $38^{\circ} 45^{\prime}-39^{\circ} 45^{\prime}$ latitudes and $30^{\circ} 15^{\prime}-$ $31^{\circ} 50^{\prime}$ longitudes in the southeast of Eskişehir province in Turkey (DSİ, 1978). The most important stream in the basin is the Sakarya River which covers 5.816.000 ha, with $6.4 \times 10^{9} \mathrm{~m}^{3}$ annual water potential and $824 \mathrm{~km}$ of length. In addition, Sakarbasi ponds consisting of East Pond $\left(2.1 \mathrm{~m}^{3} \mathrm{~s}^{-1}\right)$ and West Pond $\left(0.41 \mathrm{~m}^{3} \mathrm{~s}^{-1}\right)$ are among the most important sources of the river. The West Pond is the main water source of Cifteler Aquaculture and Research Station (CARS) which has a trout production capacity of 60 tons $\mathrm{yr}^{-1} .10$ stations were sampled (7 rivers, 2 ponds and the outlet of CARS) in April, July, September and December 2015 (Fig. 1). The coordinates of the sampling stations are given in Table 1.

Water temperature $\left({ }^{\circ} \mathrm{C}\right)$, dissolved oxygen (DO), $\mathrm{pH}$ and electrical conductivity (EC) were measured in situ using WTW Multi 3430 Set G. Sampling bottles were submerged towards the main flow of the river in the homogeneous zone to collect a sample below the surface film. Water samples were taken below the surface $(50 \mathrm{~cm}$ depth) from the ponds. At least $2 \mathrm{~L}$ of water was taken for each station per sampling season. They were then stored in iceboxes with ice packs and transferred immediately to the laboratory for later analyses. Total nitrogen (TN), orthophosphate $\left(\mathrm{PO}_{4}-\mathrm{P}\right)$ and alkalinity were measured according to APHA (2012). The results of parameters were checked against the Surface Water Quality By-Law (YSKY, 2016).

Approximately $10 \mathrm{~m}$ river stretch or pond shore was selected for diatom sampling. In rivers, riffles were selected to better indicate the variety of natural hard surfaces. Single substrate was chosen for comparability of the samples. At least five cobbles were used for sampling. However, if cobbles were not present, five small boulders were sampled 
instead. Epilithic diatoms were brushed from submerged substrates from removable cobbles and boulders approximately $10 \mathrm{~cm}^{2}$. One epilithic diatom sample was taken for one station per sampling season. The samples were fixed with ethanol solution immediately after collection (European Committee for Standardization, 2014a). Hot hydrogen peroxide method was used to clean diatom frustules in the laboratory, and slides were prepared with Naphrax (European Committee for Standardization, 2014b). Diatoms were identified with a trinocular Leica microscope and a camera using the relevant taxonomic literature (Krammer and Lange-Bertalot, 1985, 1986, 1988, 1991a, b; Hofmann et al., 2013). At least three slides per sample and 400 specimen in each slide were counted. Diatom indices were calculated with OMNIDIA 6.0.2 (Lecointe et al., 1993) and brief information was given for diatom indices in Table 2.

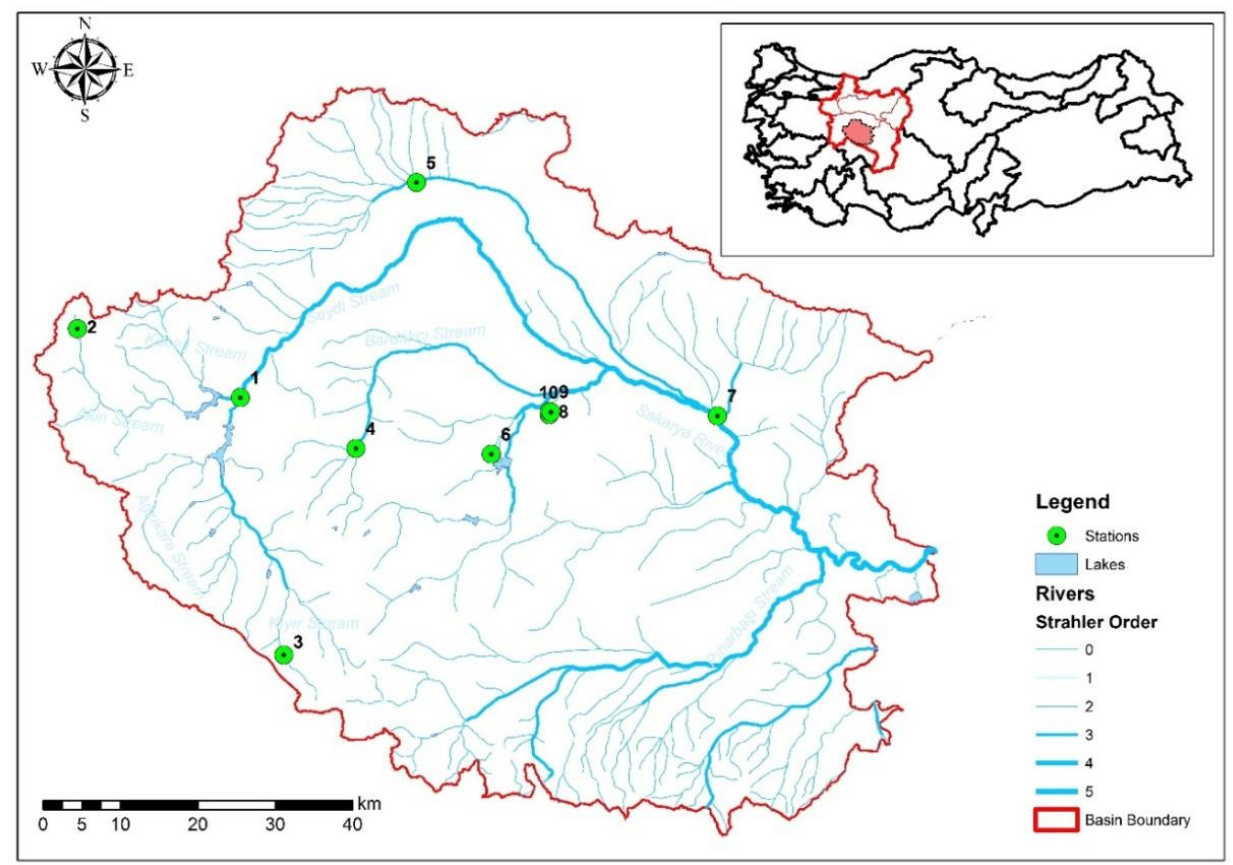

Figure 1. The Upper Sakarya Basin and sampling stations. 1: Seydisuyu river; 2: Akin river; 3: Klyır river; 4: Bardakçı river; 5: Sarısu river; 6: Ilıcabaşı river; 7: Sakarya river; 8: East pond (Sakarbasi); 9: West pond (Sakarbasi); 10: Outlet of CARS

Table 1. Coordinates of the sampling stations

\begin{tabular}{c|c|c}
\hline Stations & Water Body & Coordinates \\
\hline 1 & Seydisuyu River & $39^{\circ} 21.447^{\prime} \mathrm{N}-30^{\circ} 35.627^{\prime} \mathrm{E}$ \\
2 & Akin River & $39^{\circ} 25.794^{\prime} \mathrm{N}-30^{\circ} 20.741^{\prime} \mathrm{E}$ \\
3 & Kiyır River & $39^{\circ} 03.584^{\prime} \mathrm{N}-30^{\circ} 40.435^{\prime} \mathrm{E}$ \\
4 & Bardakçı River & $39^{\circ} 18.209^{\prime} \mathrm{N}-30^{\circ} 46.183^{\prime} \mathrm{E}$ \\
5 & Sarısu River & $39^{\circ} 36.959^{\prime} \mathrm{N}-30^{\circ} 50.732^{\prime} \mathrm{E}$ \\
6 & Ilicabaşı River & $39^{\circ} 18.155^{\prime} \mathrm{N}-30^{\circ} 58.342^{\prime} \mathrm{E}$ \\
7 & Sakarya River & $39^{\circ} 21.359^{\prime} \mathrm{N}-31^{\circ} 18.531^{\prime} \mathrm{E}$ \\
8 & East Pond (Sakarbasi) & $39^{\circ} 21.051^{\prime} \mathrm{N}-31^{\circ} 03.412^{\prime} \mathrm{E}$ \\
9 & West Pond (Sakarbasi) & $39^{\circ} 21.282^{\prime} \mathrm{N}-31^{\circ} 03.424^{\prime} \mathrm{E}$ \\
10 & Outlet of CARS & $39^{\circ} 21.233^{\prime} \mathrm{N}-31^{\circ} 03.562^{\prime} \mathrm{E}$ \\
\hline
\end{tabular}


Table 2. Information about the calculated diatom indices according to OMNIDA 6.0.2 (Lecointe et al., 1993)

\begin{tabular}{|c|c|c|c|c|}
\hline Index & Abbreviation & Reference & Scale & $\begin{array}{c}\text { Water } \\
\text { quality }\end{array}$ \\
\hline Biological Diatom Index & IBD & Coste et al., 2009 & 20 & 20 (best) \\
\hline Pollution Sensitivity Index & IPS & Cemagref, 1982 & 20 & 20 (best) \\
\hline Generic Diatom Index & IDG & Cemagref, 1982; Rumeau and Coste, 1988 & 20 & 20 (best) \\
\hline Descy Index & DESCY & Descy, 1979 & 5 & 5 (best) \\
\hline Sladecek Index & SLA & Sladecek, 1986 & 4 & 4 (worst) \\
\hline Leclercq and Maquet Index & IDSE & Leclercq and Maquet, 1987 & 5 & 5 (best) \\
\hline Artois-Picardie Diatom Index & IDAP & Prygiel et al., 1996 & 20 & 20 (best) \\
\hline Eutrophication Pollution Index-Diatoms & EPI-D & Dell’Uomo, 2004 & 4 & 4 (worst) \\
\hline Lobo Index & LOBO & Lobo et al., 2004 & 4 & 4 (worst) \\
\hline Swiss Diatom Index & DI-CH & Hürlimann and Niederhauser, 2002 & 8 & 8 (worst) \\
\hline Rott Trophic Index & TID & Rott et al., 1999 & 4 & 4 (worst) \\
\hline Rott Saprobic Index & SID & Rott et al., 1997 & 4 & 4 (worst) \\
\hline Trophic Diatom Index for Lakes & TDIL & Stenger-Kovacs et al., 2007 & 5 & 5 (best) \\
\hline Commission for Economical Community Index & CEE & Descy and Coste, 1991 & 20 & 20 (best) \\
\hline Watanabe Index & WAT & Watanabe et al., 1990 & 100 & 100 (best) \\
\hline Trophic Diatom Index & TDI & Kelly and Whitton, 1995 & 100 & 100 (worst) \\
\hline Pollution Tolerant Taxa Index & $\% \mathrm{PT}$ & Kelly and Whitton, 1995 & $\%$ & 100 (worst) \\
\hline Pampean Diatom Index & IDP & Gomez and Licursi, 2001 & 4 & 4 (worst) \\
\hline Steinberg and Schiefele Index & SHE & Steinberg and Schiefele, 1988 & 7 & 7 (best) \\
\hline
\end{tabular}

Detrended Correspondence Analysis (DCA) was performed to determine whether the ordination model was linear or unimodal before proceeding with the Canonical Correspondence Analysis (Leps and Smilauer, 2003). In order to link diatom species with water quality parameters, CCA was made with CANOCO 4.5 (ter Braak and Smilauer, 2002) and XLSTAT because the longest gradient length is above 4.0. Diatom species which were over $1 \%$ of general composition were taken into consideration. Physicochemical parameters were transformed $\ln (\mathrm{x}+1)$ except for $\mathrm{pH}$ in order to decrease skewness. CCA analysis was tested with 499 unrestricted permutations according to Monte Carlo simulation to clarify the significance of environmental variables on diatom species data. Significant variables $(P<0.01)$ were included in CCA analysis. The Pearson correlation between diatom indices and water quality parameters was calculated with XLSTAT software. In order to normalize the data, all index results were transformed to the scale $0-20$ by the OMNIDIA 6.0.2. Since orthophosphate results were lower than the detection limits $\left(<0.01 \mathrm{mg} \mathrm{L}^{-1}\right)$ in some stations, they were excluded from CCA analysis. It was performed for 39 variables (22 diatom species and 6 physicochemical parameters) in 10 stations for 4 sampling periods due to the fact that station 2 was dry in September. The seasonal variations in physicochemical parameters and community structure were analysed by using one-way variance analysis (ANOVA). Diversity and evenness of diatoms calculated for community structure.

IHF index (Fluvial Habitat Index) (Pardo et al., 2002) and MPCA Stream Habitat Assessment (MSHA) (MPCA, 2014) were used in rivers, and the ECELS index (Boix et al., 2010) was used in ponds to estimate the hydromorphological status.

Ecological status was determined according to one-out-all-out principle in the WFD (Directive, 2000). According to this principle, the lowest score of all assessment results determined the overall ecological quality class in this study. 


\section{Results}

\section{Physical and chemical water quality parameters}

In the research period, the lowest mean temperature $\left(8.53{ }^{\circ} \mathrm{C}\right)$, alkalinity (11.55 mg L $\left.\mathrm{mg}^{-1}\right)$, electrical conductivity $\left(68.73 \mu \mathrm{S} \mathrm{cm}^{-1}\right)$, total nitrogen $\left(0.50 \mathrm{mg} \mathrm{L}^{-1}\right)$ and the highest mean dissolved oxygen $\left(9.99 \mathrm{mg} \mathrm{L}^{-1}\right)$ were measured in station 2 (Table 3). $\mathrm{pH}$ ranged between 7.05 and 8.0. The highest temperature $\left(21.50^{\circ} \mathrm{C}\right)$ and total nitrogen $\left(4.06 \mathrm{mg} \mathrm{L}^{-1}\right)$ were found in station 6.

Table 3. The variation of physicochemical parameters in sampling stations by season $(n=4)$ (mean \pm standard deviation)

\begin{tabular}{|c|c|c|c|c|c|c|c|}
\hline Stations & $\begin{array}{c}\text { Temperature } \\
\left({ }^{\circ} \mathrm{C}\right)\end{array}$ & pH & 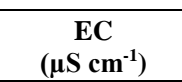 & $\begin{array}{c}\text { DO } \\
\left(\mathrm{mg} \mathrm{L}^{-1}\right)\end{array}$ & $\begin{array}{c}\mathrm{TN} \\
\left(\mathrm{mg} \mathrm{L}^{-1}\right)\end{array}$ & $\begin{array}{c}\mathrm{PO}_{4}-\mathrm{P} \\
\left(\mathrm{mg} \mathrm{L}^{-1}\right)\end{array}$ & $\begin{array}{c}\text { Alkalinity } \\
\left(\mathrm{mg} \mathrm{CaCO}_{3} \mathrm{~L}^{-1}\right)\end{array}$ \\
\hline 1 & $11.53 \pm 6.35$ & $7.68 \pm 0.28$ & $359.00 \pm 27.31$ & $9.43 \pm 1.49$ & $1.25 \pm 0.36$ & $0.03 \pm 0.04$ & $118.11 \pm 24.89$ \\
\hline 2 & $8.53 \pm 4.65$ & $7.52 \pm 0.46$ & $68.73 \pm 10.18$ & $9.99 \pm 0.83$ & $0.50 \pm 0.65$ & $0.01 \pm 0.01$ & $11.55 \pm 4.16$ \\
\hline 3 & $15.95 \pm 4.97$ & $7.58 \pm 0.15$ & $491.00 \pm 54.94$ & $8.62 \pm 1.07$ & $1.82 \pm 0.76$ & $0.03 \pm 0.05$ & $164.05 \pm 42.28$ \\
\hline 4 & $16.28 \pm 7.82$ & $8.07 \pm 0.26$ & $549.25 \pm 46.18$ & $9.00 \pm 2.11$ & $2.81 \pm 0.66$ & $0.02 \pm 0.01$ & $200.66 \pm 57.42$ \\
\hline 5 & $13.15 \pm 6.34$ & $7.56 \pm 0.17$ & $986.50 \pm 53.10$ & $4.82 \pm 2.54$ & $3.66 \pm 1.43$ & $0.01 \pm 0.01$ & $189.88 \pm 42.77$ \\
\hline 6 & $21.50 \pm 3.59$ & $7.05 \pm 0.08$ & $715.00 \pm 12.68$ & $5.39 \pm 0.27$ & $4.06 \pm 1.01$ & $0.01 \pm 0.01$ & $203.21 \pm 51.63$ \\
\hline 7 & $15.25 \pm 5.88$ & $7.72 \pm 0.36$ & $897.25 \pm 164.29$ & $7.90 \pm 1.45$ & $1.68 \pm 0.19$ & $0.03 \pm 0.02$ & $198.87 \pm 29.90$ \\
\hline 8 & $19.28 \pm 3.93$ & $7.15 \pm 0.07$ & $870.00 \pm 19.78$ & $7.14 \pm 0.58$ & $1.90 \pm 0.64$ & $0.01 \pm 0.02$ & $224.18 \pm 49.62$ \\
\hline 9 & $18.88 \pm 3.14$ & $7.17 \pm 0.10$ & $857.75 \pm 14.17$ & $7.03 \pm 0.67$ & $1.84 \pm 0.45$ & $0.01 \pm 0.00$ & $224.96 \pm 49.39$ \\
\hline 10 & $19.15 \pm 3.02$ & $7.45 \pm 0.18$ & $856.25 \pm 16.32$ & $7.06 \pm 0.63$ & $1.77 \pm 0.41$ & $0.01 \pm 0.01$ & $223.34 \pm 48.20$ \\
\hline
\end{tabular}

\section{Diatom composition}

In total, 96 diatom species which belong to 43 genera were identified from the sampling stations of the Upper Sakarya Basin (Table 4). The dominant species were Achnanthidium minutissimum, Cymbella excisa, Craticula subminuscula, Fragilaria brevistriata, Amphora pediculus, Diatoma moniliformis, Ulnaria ulna and Denticula kuetzingii. On the other hand, Cymatopleura elliptica, Caloneis bacillum, Nitzschia vitrea, Cocconeis disculus, Cymbella compacta, Cymbella parva, Gomphonema truncatum, Navicula oblonga and Pinnularia viridiformis were found rarely.

\section{Diatom indices}

The seasonal average scores of diatom indices in the sampling stations were given in Table 5. Different diatom indices revealed different water quality classes in the stations.

\section{Statistical analyses}

The seasonal variations in physicochemical parameters were found to be significant $(P<0.05)$. The unimodal ordination model was found between physicochemical parameters and diatom taxa since the longest gradient length was above 4.0. CCA analysis was performed to explain the link between physicochemical parameters and diatom taxa. The eigenvalues of the first two CCA axes were 0.792 and $0.515 .18 .8 \%$ of the cumulative variance (axis 1: 11.4\%, axis 2: 7.4) in the diatom species was explained by the first two CCA axes with $92 \%$ of the diatom species-environment correlation (Fig. 2). The amount of species-environment variance explained was 63.4\% (Table 6). The Monte Carlo test confirmed that temperature, dissolved oxygen, total nitrogen, alkalinity $(P=0.002)$ and $\mathrm{pH}(P=0.004)$ were statistically significant $(P<0.01)$. 
Table 4. Diatom composition of the sampling stations (\%)

\begin{tabular}{|c|c|c|c|c|c|c|c|c|c|c|}
\hline \multirow{2}{*}{ Species } & \multicolumn{10}{|c|}{ Composition (\%) of stations } \\
\hline & 1 & 2 & 3 & 4 & 5 & 6 & 7 & 8 & 9 & 10 \\
\hline Achnanthidium affine (Grunow) Czarnecki & 0.52 & - & - & 0.06 & 0.23 & - & - & - & - & 0.06 \\
\hline Achnanthidium minutissimum (Kützing) Czarnecki & 17.31 & - & 3.81 & 26.94 & 53.00 & 66.37 & 0.54 & 0.43 & - & 2.35 \\
\hline Achnanthidium pyrenaicum (Hustedt) H.Kobayasi & 0.35 & - & - & - & 0.17 & - & - & - & 2.86 & 0.06 \\
\hline Amphipleura pellucida (Kützing) Kützing & 0.06 & 6.07 & - & - & 0.06 & - & 0.18 & 0.12 & - & - \\
\hline Amphora copulata (Kützing) Schoeman \& R.E.M.Archibald & - & - & - & 0.12 & 0.11 & 0.30 & - & - & - & - \\
\hline Amphora ovalis (Kützing) Kützing & - & - & - & 0.24 & 0.17 & 0.18 & - & 0.06 & - & - \\
\hline Amphora pediculus (Kützing) Grunow ex A.Schmidt & 1.39 & 0.51 & 3.81 & 13.56 & 0.06 & - & 1.14 & 13.99 & 5.72 & 6.53 \\
\hline Aneumastus minor Lange-Bertalot & - & - & - & - & - & - & - & 0.25 & - & - \\
\hline Aulacoseira granulata var. angustissima (Otto Müller) Simonsen & 0.12 & - & 0.18 & - & - & - & - & - & - & - \\
\hline Bacillaria paxillifera (O.F.Müller) T.Marsson & - & - & - & - & - & - & 3.06 & - & 0.06 & - \\
\hline Brebissonia lanceolata (C.Agardh) Mahoney \& Reimer & - & - & 0.18 & - & - & - & 0.66 & - & - & - \\
\hline Caloneis bacillum (Grunow) Cleve & - & - & - & - & - & 0.12 & - & - & - & - \\
\hline Caloneis macedonica Hustedt & - & - & - & - & - & - & - & 0.25 & - & - \\
\hline Cocconeis disculus (Schumann) Cleve & - & - & - & - & - & - & - & 0.19 & - & - \\
\hline Cocconeis placentula Ehrenberg & - & 1.03 & 1.97 & 0.12 & 1.43 & 0.06 & 2.94 & 0.37 & 0.73 & - \\
\hline Cocconeis placentula var. lineata (Ehrenberg) Van Heurck & 0.29 & 2.31 & 1.97 & 0.54 & 2.34 & 0.06 & 4.92 & 0.06 & 3.71 & 0.41 \\
\hline Craticula accomoda (Hustedt) D.G.Mann & 1.91 & - & 0.66 & 0.12 & - & 0.30 & - & - & 4.81 & 0.06 \\
\hline Craticula cuspidata (Kutzing) D.G.Mann & - & - & - & - & - & - & 0.30 & - & - & - \\
\hline Craticula subminuscula (Manguin) C.E.Wetzel \& L.Ector & 16.62 & 6.92 & 40.33 & 2.29 & 0.40 & - & 2.22 & - & 0.06 & 0.24 \\
\hline Cyclostephanos dubius (Hustedt) Round & 0.41 & - & - & - & - & - & - & - & - & - \\
\hline Cyclotella atomus Hustedt & 0.29 & - & - & - & - & - & 0.06 & - & 0.06 & - \\
\hline Cyclotella distinguenda Hustedt & - & - & - & - & 7.71 & - & 0.06 & 0.25 & 4.99 & 3.12 \\
\hline Cyclotella meneghiniana Kützing & 0.29 & 0.77 & - & - & 0.29 & 0.06 & 1.86 & 0.25 & - & 0.29 \\
\hline Cymatopleura elliptica (Brébisson) W.Smith & - & - & - & 0.06 & - & - & - & - & - & - \\
\hline Cymatopleura solea (Brébisson) W.Smith & - & - & 0.06 & - & - & - & 0.30 & - & - & - \\
\hline Cymbella compacta Østrup & 0.17 & - & - & - & - & - & - & - & - & - \\
\hline
\end{tabular}


Cymbella cymbiformis C.Agardh

Cymbella excisa Kützing

Cymbella hustedtii Krasske

Cymbella lange-bertalotii Krammer

Cymbella neocistula Krammer

Cymbella parva (W.Smith) Kirchner

Cymbopleura amphicephala (Nägeli) Krammer

Denticula kuetzingii Grunow

Diatoma moniliformis (Kützing) D.M.Williams

Diatoma vulgaris Bory

Encyonema lacustre (C.Agardh) Pantocsek

Encyonema minutum (Hilse) D.G.Mann

Encyonema silesiacum (Bleisch) D.G.Mann

Encyonema ventricosum (C.Agardh) Grunow

Encyonopsis cesatii (Rabenhorst) Krammer

Encyonopsis minuta Krammer \& E.Reichardt

Epithemia turgida (Ehrenberg) Kützing

Eunotia bilunaris (Ehrenberg) Schaarschmidt

Eunotia minor (Kützing) Grunow

Fragilaria brevistriata Grunow

Fragilaria construens (Ehrenberg) Grunow

Fragilaria recapitellata Lange-Bertalot \& Metzeltin

Fragilaria vaucheriae (Kützing) J.B.Petersen

Gomphonema italicum Kützing

Gomphonema micropus Kützing

Gomphonema olivaceum (Hornemann) Brébisson

Gomphonema parvulum (Kützing) Kützing

Gomphonema subclavatum (Grunow) Grunow

Gomphonema truncatum Ehrenberg

\begin{tabular}{|c|c|c|c|c|c|c|c|c|c|}
\hline- & - & - & - & 1.77 & - & 0.12 & 0.43 & 0.49 & 0.06 \\
\hline 2.26 & 3.25 & 1.61 & 13.44 & 6.45 & 5.88 & 4.20 & 19.18 & 30.44 & 17.12 \\
\hline 0.06 & 0.26 & 0.24 & 0.30 & - & - & - & - & - & - \\
\hline 0.75 & - & - & 0.60 & - & - & 0.12 & - & - & - \\
\hline 0.06 & - & - & 0.06 & 0.46 & - & 0.84 & 0.06 & - & - \\
\hline 0.17 & - & - & - & - & - & - & - & - & - \\
\hline- & - & - & 0.06 & 0.11 & 0.36 & 1.08 & 0.43 & 0.06 & - \\
\hline 0.17 & - & - & 0.66 & 12.79 & 11.99 & 0.24 & 0.37 & 2.50 & 0.53 \\
\hline 1.16 & - & - & 15.67 & - & 0.06 & 14.27 & - & - & 4.41 \\
\hline 0.41 & - & - & 0.30 & - & - & 0.66 & 0.99 & - & 7.00 \\
\hline 0.12 & - & - & - & - & - & - & 0.12 & - & 0.06 \\
\hline 0.35 & - & - & - & - & - & - & - & - & - \\
\hline- & 1.97 & 0.48 & 0.18 & 0.06 & - & - & - & - & - \\
\hline 2.61 & 2.14 & 1.67 & 0.54 & 0.11 & - & 0.12 & - & - & - \\
\hline- & - & - & - & 0.06 & - & 0.06 & 0.12 & 0.06 & - \\
\hline 0.69 & - & 1.19 & 7.23 & 1.83 & 0.59 & 3.36 & 0.99 & 1.89 & 1.29 \\
\hline- & - & - & - & - & - & - & - & - & 0.65 \\
\hline- & 11.62 & - & - & - & - & - & - & - & - \\
\hline- & - & - & - & - & - & - & 0.25 & - & - \\
\hline 0.06 & 1.20 & - & 0.06 & 1.43 & 1.07 & 5.40 & 51.77 & 0.97 & 1.59 \\
\hline- & - & - & - & - & 0.06 & 0.18 & 0.93 & 0.30 & - \\
\hline- & 3.76 & - & - & - & - & - & - & - & - \\
\hline 0.17 & 0.26 & 0.30 & - & 0.11 & - & 0.06 & 0.06 & 3.78 & 1.29 \\
\hline- & 0.60 & 0.06 & - & - & - & 0.18 & - & 1.28 & 0.35 \\
\hline- & 18.35 & - & - & - & - & - & - & - & - \\
\hline 15.52 & 0.77 & 3.81 & 4.88 & - & - & 0.48 & - & 0.24 & 0.24 \\
\hline 4.86 & 0.77 & 9.71 & 0.48 & 1.66 & 2.08 & 0.24 & 0.06 & 4.87 & 0.18 \\
\hline- & 2.22 & - & - & - & - & - & - & - & - \\
\hline- & - & - & - & - & - & - & - & 0.18 & \\
\hline
\end{tabular}

APPLIED ECOLOGY AND ENVIRONMENTAL RESEARCH 17(4):10155-10172.

http://www.aloki.hu • ISSN 15891623 (Print) • ISSN 17850037 (Online)

DOI: http://dx.doi.org/10.15666/aeer/1704_1015510172

(c) 2019, ALÖKI Kft., Budapest, Hungary 
Halamphora veneta (Kützing) Levkov

Hantzschia amphioxys (Ehrenberg) Grunow

Hippodonta capitata (Ehrenberg) Lange-Bertalot. Metzeltin \& Witkowsk

Karayevia clevei (Grunow) Round \& Bukhtiyarova

Lindavia balatonis (Pantocsek) T.Nakov et al.

Lindavia ocellata (Pantocsek) T.Nakov et al.

Melosira varians C.Agardh

Meridion circulare (Greville) C.Agardh

Navicula antonii Lange-Bertalot

Navicula capitatoradiata H.Germain

Navicula cari Ehrenberg

Navicula cryptotenella Lange-Bertalot

Navicula kotschyi Grunow

Navicula oblonga (Kützing) Kützing

Navicula reichardtiana Lange-Bertalot

Navicula tripunctata (O.F.Müller) Bory

Nitzschia amphibia Grunow

Nitzschia capitellata Hustedt. nom. inval.

Nitzschia dissipata (Kützing) Rabenhorst

Nitzschia fonticola (Grunow) Grunow

Nitzschia frustulum (Kützing) Grunow

Nitzschia heufleriana Grunow

Nitzschia linearis W.Smith

Nitzschia recta Hantzsch ex Rabenhorst

Nitzschia vitrea G.Norman

Pinnularia viridiformis Krammer

Planothidium lanceolatum (Brébisson ex Kützing) Lange-Bertalot

Planothidium rostratum (Østrup) Lange-Bertalot

Reimeria sinuata (W.Gregory) Kociolek \& Stoermer

\begin{tabular}{|c|c|c|c|c|c|c|c|c|c}
- & - & 1.61 & 0.24 & 0.23 & - & 0.12 & - & 3.47 & 1.88 \\
0.06 & 0.85 & - & 0.24 & 0.57 & 0.06 & - & - & - & 0.12 \\
0.17 & - & - & - & - & - & 0.30 & - & - & - \\
- & 0.51 & 0.06 & - & - & - & 0.24 & 2.41 & - & - \\
1.16 & - & 0.06 & - & - & - & 1.86 & - & - & - \\
4.00 & 0.43 & 0.18 & 0.12 & - & - & 3.18 & - & 0.06 & 0.18 \\
0.58 & - & 0.06 & - & 0.74 & 0.30 & 19.85 & - & 0.85 & 0.29 \\
- & 17.52 & - & 0.18 & - & - & - & - & - & - \\
- & - & 0.71 & 0.06 & - & - & - & 0.12 & - & - \\
0.29 & - & 0.71 & 0.24 & - & - & 0.18 & - & - & - \\
- & - & - & - & - & - & - & 1.61 & - & 0.06 \\
0.17 & 0.43 & 0.30 & 1.27 & 0.17 & & 3.66 & 0.62 & 0.97 & 0.12 \\
- & - & - & - & 0.17 & 3.74 & - & 0.37 & 0.06 & - \\
- & - & - & 0.06 & - & - & 0.06 & 0.06 & - & - \\
11.70 & 2.05 & 1.19 & 1.02 & 0.11 & - & - & 0.12 & - & 0.12 \\
0.81 & - & 6.61 & 1.08 & 0.46 & - & 4.44 & - & - & 0.12 \\
1.33 & - & 1.61 & - & - & 0.12 & 1.32 & - & 0.12 & 14.47 \\
0.75 & - & 0.30 & 0.06 & - & 3.38 & 0.06 & - & 0.06 & 0.18 \\
2.95 & 0.85 & 1.43 & 4.34 & 1.77 & - & 1.92 & 0.06 & 0.24 & 0.12 \\
2.37 & 0.26 & 2.92 & - & 0.29 & - & - & - & 0.49 & 18.45 \\
- & - & 0.24 & - & 0.23 & - & - & - & - & - \\
- & - & 0.12 & 0.18 & - & - & 0.24 & - & - & - \\
- & 1.37 & 2.44 & 0.36 & 0.34 & 0.06 & 1.68 & - & 0.24 & - \\
- & - & - & - & 0.11 & - & 0.96 & - & - & - \\
- & - & - & - & - & - & 0.12 & - & - & - \\
- & - & - & - & - & - & 0.18 & - & - & - \\
- & 5.64 & 0.66 & - & 0.06 & 1.07 & - & - & - & - \\
- & - & - & - & - & - & 0.42 & - & - & - \\
0.64 & 0.26 & 0.60 & 0.24 & 0.06 & - & 0.06 & - & 0.12 & -
\end{tabular}

APPLIED ECOLOGY AND ENVIRONMENTAL RESEARCH 17(4):10155-10172.

http://www.aloki.hu • ISSN 15891623 (Print) • ISSN 17850037 (Online)

DOI: http://dx_doi org/10.15666/aeer/1704_1015510172

(c) 2019, ALÖKI Kft., Budapest, Hungary 
Rhoicosphenia abbreviata (C.Agardh) Lange-Bertalot

Rhopalodia gibba (Ehrenberg) Otto Müller

Sellaphora pupula (Kützing) Mereschkovsky

Staurosira venter (Ehrenberg) Cleve \& J.D.Möller

Stephanodiscus hantzschii Grunow

Surirella angusta Kützing

Surirella brebissoni Krammer \& Lange-Bertalot

Tryblionella apiculata W.Gregory

Tryblionella hungarica (Grunow) Frenguelli

Ulnaria acus (Kützing) Aboal

Ulnaria biceps (Kützing) Compère

Ulnaria ulna (Nitzsch) Compère

\begin{tabular}{|c|c|c|c|c|c|c|c|c|c}
0.41 & 0.51 & 0.24 & - & - & - & 1.26 & - & - & 0.06 \\
- & 0.60 & - & - & - & - & - & - & 0.18 & - \\
- & 0.43 & 0.60 & - & - & 0.12 & 0.12 & 0.12 & - & - \\
- & - & - & - & - & - & - & 0.43 & - & - \\
0.23 & 0.51 & - & - & - & - & 1.02 & - & - & 0.47 \\
0.17 & 1.54 & 0.60 & 0.24 & - & - & 1.02 & - & - & - \\
0.17 & 1.20 & 4.29 & 0.36 & 0.11 & 0.42 & 2.28 & - & 0.06 & - \\
0.23 & - & 0.36 & 0.12 & 0.11 & - & 1.20 & - & - & - \\
- & - & - & - & - & 0.59 & 0.24 & - & - & - \\
0.23 & - & - & 0.60 & 0.06 & - & 0.30 & 0.68 & 0.06 & 0.24 \\
0.23 & - & - & 0.18 & 1.09 & 0.30 & 0.18 & 1.36 & 4.57 & 6.47 \\
2.20 & 0.26 & 0.06 & 0.30 & 0.51 & 0.30 & 1.68 & 0.06 & 18.39 & 8.76
\end{tabular}

Table 5. Average scores of diatom indices in sampling stations (see abbreviations in Table 2)

\begin{tabular}{|c|c|c|c|c|c|c|c|c|c|c|c|c|c|c|c|c|c|c|c|}
\hline \multirow{2}{*}{ Stations } & IBD & IPS & IDG & DESCY & SLA & IDSE & IDAP & EPI-D & LOBO & DI-CH & TID & SID & TDIL & CEE & WAT & TDI & PT & IDP & SHE \\
\hline & 20 & 20 & 20 & 5 & 4 & 5 & 20 & 4 & 4 & 8 & 4 & 4 & 5 & 20 & 100 & 100 & $\%$ & 4 & 7 \\
\hline 1 & 13.7 & 12.7 & 12.2 & 3.50 & 2.03 & 3.11 & 11.6 & 1.75 & 2.11 & 4.15 & 2.81 & 2.39 & 2.96 & 11.4 & 58.30 & 77.23 & 26.3 & 1.80 & 4.35 \\
\hline 2 & 15.9 & 14.3 & 14.9 & 4.29 & 1.27 & 3.75 & 11.0 & 1.26 & 2.00 & 3.97 & 2.85 & 1.97 & 2.76 & 15.2 & 61.93 & 52.46 & 29.5 & 1.13 & 5.09 \\
\hline 3 & 10.1 & 10.0 & 9.6 & 3.73 & 2.20 & 2.81 & 8.7 & 2.41 & 2.02 & 5.97 & 3.34 & 2.64 & 1.83 & 10.0 & 39.69 & 84.74 & 57.3 & 2.20 & 3.33 \\
\hline 4 & 19.1 & 16.0 & 14.8 & 4.50 & 1.41 & 4.09 & 16.1 & 1.20 & 3.33 & 3.33 & 2.23 & 2.03 & 3.53 & 15.4 & 72.77 & 51.03 & 3.6 & 1.49 & 5.47 \\
\hline 5 & 18.8 & 16.8 & 16.1 & 3.97 & 1.22 & 4.14 & 17.6 & 0.76 & 3.64 & 2.90 & 1.64 & 1.46 & 3.53 & 16.0 & 79.02 & 42.09 & 3.4 & 1.29 & 5.86 \\
\hline 6 & 18.2 & 15.6 & 16.0 & 3.85 & 1.23 & 4.21 & 18.8 & 0.86 & 3.98 & 3.33 & 1.81 & 1.58 & 3.84 & 15.4 & 83.24 & 38.15 & 6.3 & 1.21 & 5.72 \\
\hline 7 & 15.6 & 13.3 & 12.6 & 3.94 & 1.67 & 3.45 & 13.0 & 1.84 & 3.08 & 4.23 & 2.77 & 2.06 & 2.93 & 11.5 & 54.64 & 71.34 & 6.3 & 1.98 & 4.96 \\
\hline 8 & 15.9 & 13.1 & 13.9 & 4.80 & 1.48 & 3.87 & 15.2 & 1.44 & 2.79 & 3.33 & 2.79 & 1.50 & 2.98 & 14.7 & 58.73 & 73.22 & 2.5 & 1.73 & 5.45 \\
\hline 9 & 14.7 & 12.0 & 14.5 & 3.02 & 1.82 & 3.16 & 7.7 & 1.72 & 2.65 & 4.88 & 3.07 & 2.08 & 2.57 & 11.9 & 54.25 & 63.62 & 9.3 & 1.95 & 5.21 \\
\hline 10 & 15.0 & 12.2 & 10.4 & 3.53 & 1.64 & 3.35 & 9.2 & 1.75 & 2.65 & 4.91 & 3.24 & 2.22 & 3.34 & 11.7 & 64.34 & 72.57 & 20.2 & 1.86 & 5.37 \\
\hline
\end{tabular}

APPLIED ECOLOGY AND ENVIRONMENTAL RESEARCH 17(4):10155-10172.

http://www aloki.hu • ISSN 15891623 (Print) • ISSN 17850037 (Online)

DOI: http://dx.doi.org/10.15666/aeer/1704_1015510172

๑) 2019, ALÖKI Kft., Budapest, Hungary 


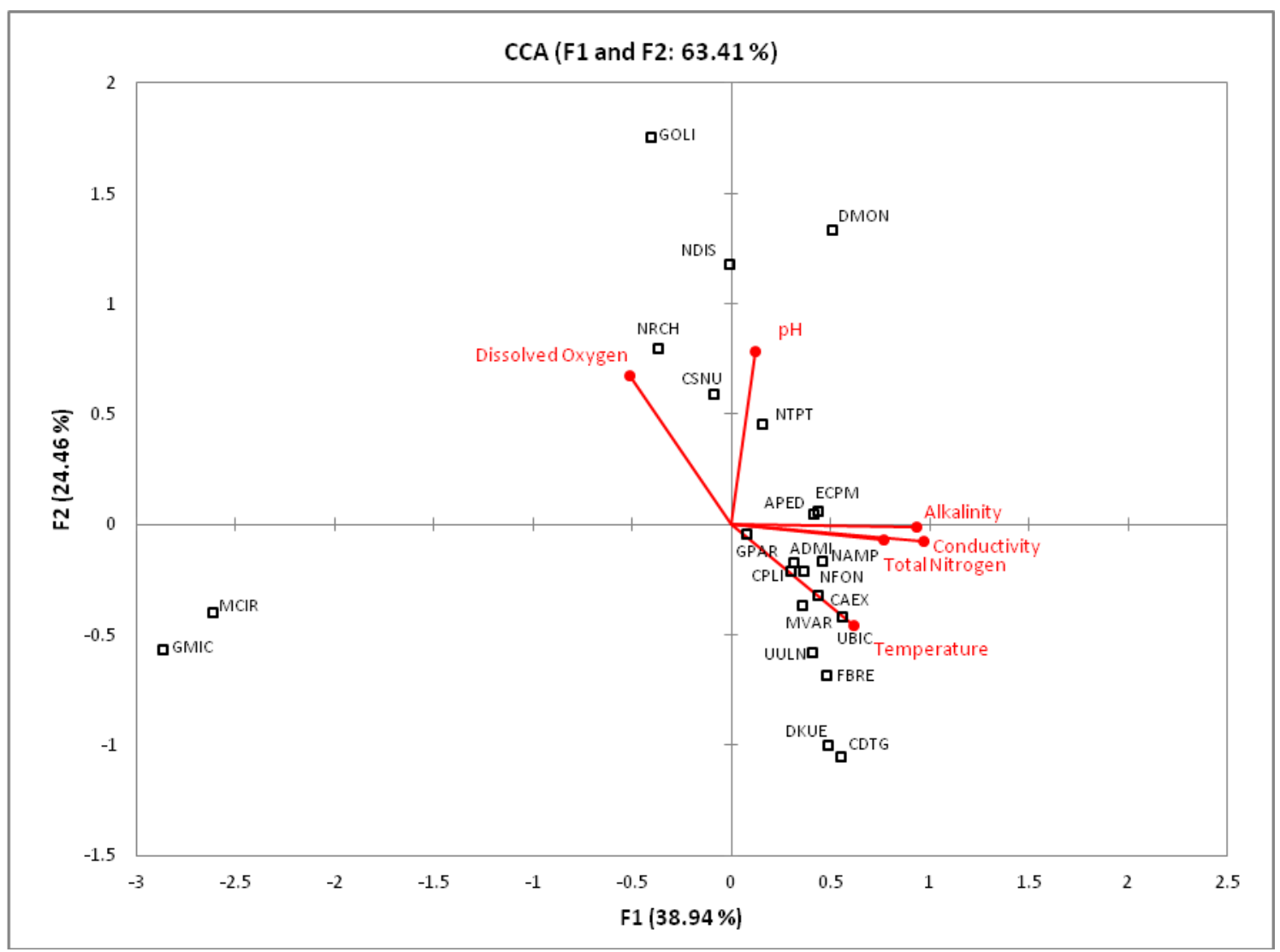

Figure 2. CCA plot of species and physicochemical parameters in sampling stations. ADMI, Achnanthidium minutissimum; APED, Amphora pediculus; CPLI, Cocconeis placentula var. lineata; CSNU, Craticula subminuscula; CDTG, Cyclotella distinguenda; CAEX, Cymbella excisa; DKUE, Denticula kuetzingii; DMON, Diatoma moniliformis; ECPM, Encyonopsis minuta; FBRE, Fragilaria brevistriata; GMIC, Gomphonema micropus; GOLI, Gomphonema olivaceum; GPAR, Gomphonema parvulum; MVAR, Melosira varians; MCIR, Meridion circulare; NRCH, Navicula reichardtiana; NTPT, Navicula tripunctata; NAMP, Nitzschia amphibia; NDIS, Nitzschia dissipata; NFON, Nitzschia fonticola; UBIC, Ulnaria biceps; UULN, Ulnaria ulna

Table 6. Summary of canonical correspondence analysis for diatom species and physicochemical parameters

\begin{tabular}{l|c|c|c|c|c}
\hline \multicolumn{1}{c|}{ Axes } & $\mathbf{1}$ & $\mathbf{2}$ & $\mathbf{3}$ & $\mathbf{4}$ & Total inertia \\
\hline Eigenvalues & 0.792 & 0.515 & 0.384 & 0.199 & 6.961 \\
Species-environment correlations & 0.954 & 0.920 & 0.888 & 0.711 & \\
Cumulative percentage varians of species data & 11.4 & 18.8 & 24.3 & 27.1 & \\
of species environment relation & 38.9 & 63.4 & 81.2 & 90.8 & \\
Sum of all eigenvalues & & & & & 6.961 \\
Sum of all canonical eigenvalues & & & & & 2.080 \\
\hline
\end{tabular}

Figure 2 shows the CCA biplot of physicochemical parameters and diatom species for the first two axes. As shown in Figure 2, there is a negative relation between 
temperature and dissolved oxygen whereas a positive relation was found between alkalinity and conductivity. Gomphonema micropus and Meridion circulare were found to be in a separate quadrat from the other species and physicochemical parameters. Conductivity, alkalinity, total nitrogen and temperature were the parameters playing an important role in the distribution of species. When the clusters of species were examined, a large percentage of the species were clustered around temperature. In terms of nutrients, total nitrogen showed a close relation with temperature in the distribution of species. Pearson correlations between physicochemical parameters and diatom indices were shown in Table 7.

Table 7. Pearson correlations between physicochemical parameters and diatom indices $(n=39)$ (see abbreviations in Table 2)

\begin{tabular}{c|c|c|c|c|c|c}
\hline & Temperature & Dissolved oxygen & $\mathbf{p H}$ & Conductivity & Alkalinity & $\begin{array}{c}\text { Total } \\
\text { nitrogen }\end{array}$ \\
\hline IBD & -0.2319 & -0.1942 & -0.0253 & 0.1525 & 0.0380 & 0.1824 \\
IPS & $\mathbf{- 0 . 3 7 5 9 *}$ & -0.1402 & 0.0327 & 0.0522 & -0.0699 & 0.1537 \\
IDG & -0.2359 & -0.2117 & -0.1855 & 0.0115 & -0.0778 & 0.1581 \\
DESCY & -0.2324 & 0.1304 & 0.0363 & -0.0646 & -0.0912 & -0.0181 \\
SLA & -0.3058 & -0.1922 & -0.2026 & 0.0194 & -0.1307 & 0.0256 \\
IDSE & -0.2704 & -0.1818 & -0.1078 & 0.0974 & -0.0320 & 0.1812 \\
IDAP & -0.1688 & $\mathbf{- 0 . 3 1 9 2 *}$ & -0.0505 & 0.1989 & 0.1029 & $\mathbf{0 . 4 0 0 1}$ \\
EPI-D & $\mathbf{- 0 . 3 2 6 6 *}$ & -0.2527 & -0.1503 & 0.0591 & -0.0811 & 0.1614 \\
LOBO & 0.0603 & $\mathbf{- 0 . 3 8 6 9 *}$ & -0.1503 & $\mathbf{0 . 3 6 7 1 *}$ & 0.2625 & $\mathbf{0 . 3 8 1 1 *}$ \\
DI-CH & $\mathbf{- 0 . 3 6 8 0 *}$ & -0.0799 & -0.0692 & 0.0647 & -0.0363 & 0.0966 \\
TID & -0.1553 & $\mathbf{- 0 . 3 7 8} *$ & -0.0525 & 0.1685 & 0.0917 & $\mathbf{0 . 4 5 1 3} *$ \\
SID & -0.0618 & $\mathbf{- 0 . 4 4 5 7 * *}$ & $\mathbf{- 0 . 3 6 7 7 *}$ & 0.2452 & 0.0950 & 0.2171 \\
TDIL & -0.2510 & -0.1764 & -0.0224 & 0.2013 & 0.0946 & 0.2741 \\
CEE & -0.3051 & -0.1447 & -0.1365 & -0.0180 & -0.1265 & 0.1088 \\
WAT & -0.2061 & -0.2569 & -0.1052 & 0.1341 & 0.0211 & 0.2587 \\
TDI & -0.1591 & -0.2998 & -0.0750 & 0.0050 & -0.1057 & 0.3071 \\
\% PT & 0.0783 & 0.2654 & 0.1024 & $\mathbf{- 0 . 3 8 3 7 *}$ & -0.2226 & -0.2390 \\
IDP & $\mathbf{- 0 . 4 2 6 4 * *}$ & -0.1075 & 0.0793 & -0.2300 & $\mathbf{- 0 . 3 2 2 4 *}$ & 0.0087 \\
SHE & -0.1191 & -0.2922 & -0.2020 & 0.2243 & 0.0750 & 0.1483 \\
\hline
\end{tabular}

$* P<0.05, * * P<0.01$

The IDP index which has the highest correlation with temperature and which plays an essential role in the distribution of species, was used to evaluate the diatom results. IPS, DI-CH and EPI-D indices were also correlated with temperature respectively after IDP index. The IDP index results regressed against total nitrogen which also affected the distribution of diatom species (Fig. 2). Station 2 is excluded from the regression since the species were found to be in completely different quadrats.

\section{Hydromorphological results}

The hydromorphological quality in the sampling stations varied between high and moderate (Table 8). Station 2 is in high hydromorphological status, whereas stations 5, 6 and 10 are in moderate hydromorphological status. 
Table 8. Hydromorphological status in sampling stations

\begin{tabular}{c|c|c|c|c}
\hline Stations & MSHA Score & IHF Score & ECELS Score & Status \\
\hline 1 & 62 & 65 & - & Good \\
2 & 82 & 82 & - & High \\
3 & 71 & 68 & - & Good \\
4 & 61 & 66 & - & Good \\
5 & 54 & 58 & - & Moderate \\
6 & 49 & 56 & - & Moderate \\
7 & 61 & 67 & - & Good \\
8 & - & - & 71 & Good \\
9 & - & - & 70 & Good \\
10 & 45 & 52 & - & Moderate \\
\hline
\end{tabular}

\section{Ecological status}

Ecological status was classified based on physicochemical parameters, biological results obtained by IDP index and hydromorphological findings (Table 9).

Table 9. Ecological status in sampling stations

\begin{tabular}{c|c|c|c|c|c}
\hline Stations & $\begin{array}{c}\text { Physicochemical } \\
\text { status }\end{array}$ & $\begin{array}{c}\text { Biological } \\
\text { index results }\end{array}$ & $\begin{array}{c}\text { Biological } \\
\text { status }\end{array}$ & $\begin{array}{c}\text { Hydromorphological } \\
\text { status }\end{array}$ & $\begin{array}{c}\text { Ecological } \\
\text { status }\end{array}$ \\
\hline $\mathbf{1}$ & I & 1.80 & II & II & II \\
$\mathbf{2}$ & I & 1.13 & I & I & I \\
$\mathbf{3}$ & II & 2.20 & III & II & III \\
$\mathbf{4}$ & II & 1.49 & I & II & II \\
$\mathbf{5}$ & III & $1.29 *$ & I* & III & III \\
$\mathbf{6}$ & III & $1.21^{*}$ & I* & III & III \\
$\mathbf{7}$ & II & 1.98 & II & II & II \\
$\mathbf{8}$ & II & 1.73 & II & II & II \\
$\mathbf{9}$ & II & 1.95 & II & II & II \\
$\mathbf{1 0}$ & II & 1.86 & II & III & . \\
\hline
\end{tabular}

*Given that Achnanthidium minutissimum which is found in stations 5 and 6 dominantly is excluded from the assessment, station 5 is found to be in class II (1.79) and station 6 is found to be in class III (2.04) according to IDP results

\section{Discussion}

\section{Distribution of diatom species}

In this study, Achnanthidium minutissimum, Cymbella excisa, Craticula subminuscula and Fragilaria brevistriata were determined as dominant species. The most dominant species, Achnanthidium minutissimum, is reported as a problematic both from taxonomic and ecological perspectives (Potapova, 2007). Kelly and Whitton (1995) stated that this species is dominant in high-altitude regions and in oligomesotrophic rivers. According to Steinberg and Schiefele (1988), it is a species that avoids conditions worse than $\beta$-mesosaprobic. In addition, according to Cox (1996), it is described as a species which commonly occurs in waters with a wide range of quality, 
but which is sensitive to waste water and $\beta$ - $\alpha$-mesosaprobic conditions. In the present study, Achnanthidium minutissimum was found to be a dominant species in some stations with diverse water quality. This might result from the fact that this species occurs in wide quality range from clean to polluted waters.

\section{Statistical analyses and diatom index}

In this study, most of the species clustered around the temperature. Toman et al. (2014) reported that temperature is listed among critical parameters for diatom composition which covers Achnanthidium minutissimum as a dominant species. Similarly, Izagirre and Elosegi (2005) determined that temperature was a parameter which affected diatom composition.

CCA showed that station 2 revealed the greatest differences compared to the other stations. Station 2 could only be sampled three times since it was dry in September, and it was observed that the results of these three sampling campaigns were concentrated in a different quadrat from other stations. Türkmen Mountain, with an altitude of $1829 \mathrm{~m}$, is the highest mountain in Eskişehir province (ÇSB, 2014). Solak ve Wojtal (2012) identified 304 species in a study conducted on that mountain, and described 59 species out of 304 as the most common ones for Turkey, on the other side which can occur in completely different water bodies. According to Levkov et al. (2013), new species such as Luticola kemalii, Luticola angusta and Luticola rotunda were identified in samples taken from Türkmen Mountain. The number of species in station 2 was found higher than the other stations.

When examining the correlations between the indices and temperature which affects the distribution of the large percentage of species, it was found out that the highest correlation is obtained with IDP $(r=-0.4264, P<0.01)$ index. The IDP index has also a correlation in the distribution of species with alkalinity which varies significantly $(\mathrm{r}=$ $0.3224, P<0.05)$. A significant correlation was found between the index results of IDP, which is an eutrophication index, and $\log$ total nitrogen $\left(\mathrm{R}^{2}=0.7091\right)$. As a result, IDP index was used in the evaluation of diatoms in the Upper Sakarya Basin.

The IDP index was developed for rivers located in the Pampean Plain of Argentina. Sensitivity values of 210 diatom species against organic richness and eutrophication were determined taking into account $\mathrm{BOD}_{5}, \mathrm{NH}^{+}$and $\mathrm{PO}^{-3}$ (Gomez and Licursi, 2001). The IDP index was first used in Turkey by Kalyoncu et al. (2009) to determine the water quality of the Aksu River. In that study, the IDP index and $\mathrm{NH}_{4}^{+}(\mathrm{r}=0.854$, $P<0.01)$ provided the highest correlation between diatom indices and physicochemical parameters.

\section{Ecological status}

Concerning chemical, biological and hydromorphological water quality, station 2 was estimated to be in class I according to Surface Water Quality By-law, IDP index and hydromorphological assessment respectively. According to one-out-all-out principle of WFD, the ecological status of station 2 is estimated as class I. Since the station 2 is located far away from pressures and species diversity of this station is different from the other stations, monitoring effort should be sustained in here. It is the only station in this study with a high hydromorphological status, which might be a reference site. 
Stations 5 and 6 were estimated to be in class III chemical water quality according to the Surface Water Quality By-law, class I biological water quality according to the IDP index and class III according to hydromorphological assessments. This two-class difference between chemical and biological classification was only observed in these stations. According to one-out-all-out principle, the ecological status of both stations was estimated as class III. Both stations are fed by groundwater and there are agricultural areas in the vicinity. In addition, Achnanthidium minutissimum was the dominant species in each station. When these findings were tried to be confirmed by different indices, it was found out that stations 5 and 6 are categorized in class I water quality according to the IBD index but class $\mathrm{V}$ water quality according to the LOBO index. For this reason, station 5 is categorized in class II water quality (1.79) and station 6 is categorized in class III water quality (2.04) according to IDP index when Achnanthidium minutissimum is excluded from the assessment.

Station 10 is categorized in class II chemical and biological water quality according to the Surface Water Quality By-law and IDP index respectively, and in class III water quality according to hydromorphological assessment. According to one-out-all-out principle, the ecological status of station 10 was estimated as class II. Station 10 is located on a small creek where the effluents of CARS -which is fed by waters coming from station 9- confluence with the Sakarya River. However, chemical and biological classification results do not differ too much between stations 9 and 10. Only the diatom composition differs between the stations but this difference did not have any implications in the index results. Kirkağaç et al. (2009) stated that the invertebrate abundance in the inlet water was lower than the outlet water of CARS. However, the composition did not differ and only the Gastropoda species which are tolerant to pollution were found in the inlet and outlet water. As a result of this study, diatoms are concluded as important biological indicators for the monitoring the effluents of fish farms.

Generally, the results of chemical and biological assessment were similar for all stations. The results of five stations $(2,7,8,9,10)$ were observed in the same quality class and there was one class difference among three stations $(1,3,4)$. Two-class quality difference was only observed in stations 5 and 6 , and the dominant species in those stations is Achnanthidium minutissimum, which is problematic in terms of taxonomy and ecology. According to the results of IDP index, which is calculated leaving aside this species, there is only one-class quality difference between chemical and biological status in station 5, while the same results were obtained in station 6 .

According to a study conducted by Kalyoncu et al. (2004) in Ağlasun Stream, two different water quality classes were identified in terms of physicochemical parameters and epilithic algae. Water quality was then classified based on epilithic algae, which positively deviated half water quality class. In another study, Kalyoncu (2006) reported that water quality assessment was made according to epilithic diatoms, which positively deviated half water quality class from the assessment based on the results of physicochemical parameters. The differences between physicochemical and biological quality assessment observed in this study conducted in the Upper Sakarya Basin reveal similarities with the studies mentioned above. 


\section{Conclusions}

As a summary, it is essential to develop biological indices for ecological status classification for water resources. Turkey is a very rich country in terms of biological diversity because of its geographical position. This diversity varies from region to region, and it affects the application of biological indices. For this reason, it might be recommended that eco-regions should be determined for monitoring of aquatic organisms, and the class boundaries of biological indices should be developed for these eco-regions.

Biological monitoring is carried out in river basins throughout Turkey. In this study, ecological status was estimated based on diatoms which are used as biological quality elements in rivers and ponds. The findings of this study may serve as an example both for water quality monitoring and management in Turkey. Sakarbasi Springs, which are the source of the Sakarya River, are used for several purposes, such as agriculture, recreation and aquaculture. It is therefore important to monitor these water bodies for controlling the pollution of the river.

In conclusion, it is necessary to take action for water bodies which are in moderate ecological status according to this study, and water bodies in good ecological status should be maintained. The Upper Sakarya Basin was monitored within the scope of the reference monitoring network since it forms the headwaters of the Sakarya Basin and has a high water potential. Station 2, Akin River which is in high ecological status might be proposed as a reference site for mountainous rivers with low alkalinity.

Acknowledgements. This study is a part of doctoral thesis "The Use of Phytobenthos for the Ecological Status Assessment in Upper Sakarya Basin" which was prepared in Turkey.

\section{REFERENCES}

[1] APHA (2012): Standard Methods for the Examination of Water and Wastewater. $22^{\text {nd }}$ Ed. - American Public Health Association, Washington.

[2] Atıc1, T., Tokatlı, C., Çiçek, A. (2018): Diatoms of Seydisuyu stream basin (Turkey) and assessment of water quality by statistical and biological approaches. - Sigma Journal of Engineering and Natural Sciences 36(1): 271-288.

[3] Boix, D., Caiola, N., Canedo-Argüelles, M., Gascon, S., Ibanez, C., Nebra, A., Quintana, X. D., Rieradevall, M., Sala, J., Sanchez-Millaruelo, N., Sola, C., Munne, A. (2010): Avaluacio de l'estat ecologic de les zones humides i ajust dels indicadors de qualitat. Generalitat de Catalunya: Agencia Catalana de l'Aigua, Departament de Medi Ambient i Habitatge, Barcelona.

[4] Cemagref (1982): Etude de Methodes Biologiques Quantitatives d'Appreciation de la Qualit des Eaux. - A.F.B. Rhône-Mediterrannee-Corse, Lyon.

[5] Coste, M., Boutry, S., Tison-Rosebery, J., Delmas, F. (2009): Improvements of the biological diatom index (BDI): Description and efficiency of the new version (BDI2006). - Ecological Indicators 9: 621-650.

[6] Cox, E. J. (1996): Identification of Freshwater Diatoms from Live Material. - Chapman and Hall, London.

[7] ÇŞB (2014): Kütahya Province Environment Report. - ÇŞB, Kütahya (in Turkish).

[8] Dell'Uomo, A. (2004): L'indice diatomico di eutrofizzazione/polluzione (EPI-D) nel monitoraggio delle acque correnti. Lince guida. - APAT Agenzia per la protezione dell'ambiente e per I servizi tecnici, Roma. 
[9] Demir, N., Çetin, T., Gök, C., Şanal, M. (2017): First biological monitoring in the Akarçay Basin according to the Water Framework Directive: phytoplankton and phytobenthos. - Turkish Journal of Water Science and Management 1: 90-107.

[10] Descy, J. P. (1979): A new approach to water quality estimation using diatoms. - Nowa Hedwigia 64: 305-323.

[11] Descy, J. P., Coste, M. (1991): A test of methods for assessing water quality based on diatoms. - Verhandlungen der Internationalen Vereinigung Für Theorestische und Angewandte Limnologie 24: 2112-2116.

[12] Directive (2000): Directive 2000/60/EC of the European parliament and of the council of 23 October 2000 establishing a framework for community action in the field of water policy. - O. J. E. C. 327: 1-72.

[13] DSİ (1978): Upper Sakarya Basin Hydrogeological Investigation Report. - DSİ Basım ve Foto-film İşletme Müdürlüğü Matbaası, Ankara (in Turkish).

[14] European Committee for Standardization (2014a): Water quality - guidance standard for the routine sampling and preparation of benthic diatoms from rivers and lakes. European Standard EN 13946, Brussels.

[15] European Committee for Standardization (2014b): Water quality - guidance standard for the identification and enumeration of benthic diatoms from rivers and lakes. - European Standard EN 14407, Brussels.

[16] Gómez, N., Licursi, M. (2001): The Pampean Diatom Index (IDP) for assessment of rivers and streams in Argentina. - Aqua. Ecol. 35: 173-181.

[17] Hofmann, G., Werum, M., Lange-Bertalot, H. (2013): Diatomeen im Süßwasserbenthos von Mitteleuropa. - Koeltz Scientific Books, Königstein.

[18] Hürlimann, J. Niederhauser, P. (2002): Methode d'etude et d'appreciation de l'etat de santé des cours d'eau: Diatomees-niveau R (region). - OFEFP, Berne.

[19] Izagirre, O., Elosegi, A. (2005): Environmental control of seasonal and inter-annual variations of periphytic biomass in a North Iberian stream. - Ann. Limnol.-Int. J. Lim. 41(1): 35-46.

[20] Kalyoncu, H. (2006): Determination of water quality in Isparta stream according to physicochemical parameters and epilithic diatoms. - S. D. Ü. Fen Edebiyat Fakültesi, Fen Dergisi 1(1-2): 14-25 (in Turkish).

[21] Kalyoncu, H., Barlas, M., Ertan, Ö. O., Gülbay, H. (2004): Determination of water quality of Ağlasun stream according to physicochemical parameters and epilithic algae. S. D. Ü. Eğirdir Su Ürünleri Dergisi 2(12): 7-14 (in Turkish).

[22] Kalyoncu, H., Barlas, M., Ertan, Ö. O. (2009): The study of the water quality of the Aksu stream according to the biotic index (diatoms and invertebrates) and physic-chemical parameters, the relations of the organisms with the water quality. - Türk Bilim Araştırma Vakfi 2: 45-57 (in Turkish).

[23] Kelly, M. (2013): Data rich, information poor? Phytobenthos assessment and the Water Framework Directive. - European Journal of Phycology 48: 437-450.

[24] Kelly, M. G., Whitton, B. A. (1995): The trophic diatom index: a new diatom index for monitoring eutrophication in rivers. - J. Appl. Phycol. 7: 433-444.

[25] Kırkağaç, M. Ü., Pulatsü, S., Topçu, A. (2009): Trout farm effluent effects on water sediment quality and benthos. - Clean 37(4-5): 386-391.

[26] Kivrak, E., Uygun, A., Kalyoncu, H. (2012): Application of diatom indices to assess water quality of the Akarçay stream (Afyonkarahisar, Türkiye). - AKU J Sci 12: 27-38 (in Turkish).

[27] Krammer, K., Lange-Bertalot, H. (1985): Naviculaceae. Bibliotheca Diatomologia, Band 9. - J. Cramer, Berlin-Stuttgart.

[28] Krammer, K., Lange-Bertalot, H. (1986): Bacillariophyceae. 1. Teil: Naviculaceae. - In: Ettl, H., Gerloff, J., Heynig, H., Mollenhauer, D. (eds.) Süsswasser Flora von Mitteleuropa. Band 2/1. Gustav Fischer Verlag, Stuttgart. 
[29] Krammer, K., Lange-Bertalot, H. (1988): Bacillariophyceae. 2. Teil: Bacillariaceae, Epithemiaceae, Surirellaceae. - In: Ettl, H., Gerloff, J., Heynig, H., Mollenhauer, D. (eds.) Süsswasserflora von Mitteleuropa, Band 2/2. VEB Gustav Fischer Verlag, Jena.

[30] Krammer, K., Lange-Bertalot, H. (1991a): Bacillariophyceae. 3. Teil: Centrales, Fragilariaceae, Eunotiaceae. - In: Ettl, H., Gerloff, J., Heynig, H., Mollenhauer, D. (eds.) Süsswasserflora von Mitteleuropa, Band 2/3. Gustav Fischer Verlag, Stuttgart, Jena.

[31] Krammer, K., Lange-Bertalot, H. (1991b): Bacillariophyceae. 4. Teil: Achnanthaceae, Kritische Ergänzungen zu Navicula (Lineolatae) und Gomphonema, Gesamtliteraturverzeichnis. Teil 1-4. - In: Ettl, H., Gärtner, G., Gerloff, J., Heynig, H., Mollenhauer, D. (eds.) Süsswasserflora von Mitteleuropa, Band 2/4. Gustav Fischer Verlag, Stuttgart, Jena.

[32] Leclercq, L., Maquet, B. (1987): Deux Nouveaux Indices Chimiques et Diatomiques de Qualite d'eau Courante. Application au Samson et Ses Affluents (Bassin de la Meuse Belge). Comparison Avec d'autres Indices Chimiques Biocenotiques et Diatomiques. Institute Royal des Sciences Naturelles de Belgique, Document de Travail 38: 1-113.

[33] Lecointe, C., Coste, M., Prygiel, J. (1993): "Omnidia": software for taxonomy, calculation of diatom indices and inventories management. - Hydrobiologia 269/270: 509-513.

[34] Lenoir, A., Coste, M. (1996): Development of a Practical Diatom Index of Overall Water Quality Applicable to the French National Water Board Network. - In: Whitton, B. A., Rott, E. (eds.) Use of Algae for Monitoring Rivers II. Studia, Innsbruck, pp. 29-43.

[35] Leps, J., Smilauer, P. (2003): Multivariate Analysis of Ecological Data Using CANOCO. - Cambridge University Press, Cambridge.

[36] Levkov, Z., Metzeltin, D., Pavlov, A. (2013): Luticola and Luticolopsis. - In: LangeBertalot, H. (eds.) Diatoms of Europe, Diatoms of the European Inland Waters and Comparable Habitats. Vol. 7. Koeltz Scientific Books, Konigstein.

[37] Lobo, E. A., Bes, D., Tudesque, L. Ector, L. (2004): Water quality assessment of the Pardinho River, RS, Brazil, using epilithic diatom assemblages and faecal coliforms as biological indicators. - Vie et Milieu/Life and Environment 54: 115-125.

[38] Morkoyunlu Yüce, A., Gönülol, A. (2016): Evaluations of epilithic diatoms and biotic index in Sakarya River, Turkey. - Pak. J. Bot 48(5): 2153-2158.

[39] MPCA (2014): MPCA stream habitat assessment (MSHA) protocol for stream monitoring sites. - Minnesota Pollution Control Agency. Minnesota, USA.

[40] Muxika, I., Borja, A., Bald, J. (2007): Using historical data, expert judgement and multivariate analysis in assessing reference conditions and benthic ecological status, according to the European Water Framework Directive. - Marine Pollution Bulletin 55: 16-29.

[41] Pardo, I., Alvarez, M., Casas, J., Moreno, J. L., Vivas, S., Bonada, N., Alba-Tercedor, J., Jaimez-Cuellar, P., Moya, G., Prat, N., Robles, S., Suarez, M. L., Toro, M., VidalAbarca, M. R. (2002): El habitat de los rios mediterraneos. Diseno de un indice de diversidad de habitat. - Limnetica 21: 115-133.

[42] Potapova, M. (2007): Morphological and ecological variation within the Achnanthidium minutissimum (Bacillariophyceae) species complex. - J. Phycol. 43: 561-575.

[43] Prygiel, J., Leveque, L., Iserentant, R. (1996): L'IDP: Un nouvel indice diatomique pratique pour l'evaluation de la qualite des eaux en reseau de surveillance. - Revue des Sciences de I'eau 9: 97-113.

[44] Rott, E., Hofmann, G., Pall, K., Pfister, P. Pipp, E. (1997): Indikationslisten für Aufwuchsalgen in Österreichischen Fliessgewässern. Teil 1: Saprobielle Indikation. Bundeministeriums für Land- und Forstwirtschaft, Wien.

[45] Rott, E., Pipp, E., Pfister, P., Van Dam, H., Ortler, K., Binder, N., Pall, K. (1999): Indikationslisten für Aufwuchsalgen in österreichischen Fliessgewässern. Teil 2: Trophieindikation (sowie geochemische Präferenzen, taxonomische und toxikologische 
Anmerkungen). - Wasserwirtschaftskataster, Bundesministerium f. Land- u. Forstwirtschaft, Wien.

[46] Rumeau, A. Coste, M. (1988): Initiation à la systématique des diatomées d'eau douce. Bull. Fr. Piscic. 309: 69.

[47] Sladecek, V. (1986): Diatoms as indicators of organic pollution. - Acta Hydroch Hydrobiol. 14: 555-566.

[48] Solak, C. N., Wojtal, A. Z. (2012): Diatoms in spring and streams of Türkmen Mt. (Sakarya River Basin) common in Turkish inland waters. - Polish Botanical Journal 57(2): 375-425.

[49] Solak, C. N., Barinova, S., Acs, E., Dayığlu, H. (2012): Diversity and ecology of diatoms from Felenk creek (Sakarya River basin) Turkey. - Turk J Bot. 36: 191-203.

[50] Steinberg, C., Schiefele, S. (1988): Biological indication of trophy and pollution of running waters. - Z. Wasser. Abwasser. Forsch. 21: 227-234.

[51] Stenger-Kovacs, C., Buczko, K., Hajnal, E. Padisak, J. (2007): Epiphytic, littoral diatoms as bioindicators of shallow lake trophic status: Trophic Diatom Index for Lakes (TDIL) developed in Hungary. - Hydrobiologia 589: 141-154.

[52] ter Braak, C. J. F., Milauer, P. (2002): CANOCO Reference Manual and CanoDraw for Windows User's Guide: Software for Canonical Community Ordination (Version 4.5). Microcomputer Power Press, Ithaca.

[53] Tokatl1, C. (2012): Use of some diatom indices for evaluating water quality: Sample of Gürleyik stream (Eskişehir). - Dumlupınar Üniversitesi Fen Bilimleri Enstitüsü Dergisi 29: 21-28 (in Turkish).

[54] Toman, J. M., Groseli, A. M., Zelnik, I. (2014): The influence of selected factors on the distribution of epilithic diatoms in a torrential river the Kamniska Bistrica (Slovenia). Acta Bot. Croat. 73(2): 447-463.

[55] Toudjani, A. A., Çelekli, A., Gümüş, E. Y., Kayhan, S., Lekesiz, H. Ö., Çetin, T. (2017): A new diatom index to assess ecological quality of running waters: a case study of water bodies in western Anatolia. - Int. J. Lim, 53: 333-343.

[56] Watanabe, T., Asai, K. Houki, A. (1990): Numerical Simulation of Organic Pollution in Flowing Waters. - In: Cheremisinoff, P. N. (ed.) Encyclopedia of Environmental Control Technology. Vol. 4. Hazardous Waste Containment and Treatment. Gulf Publishing Company, Houston, pp. 251-284.

[57] YSKY (2016): Surface Water Quality By-Law. - T. C. Orman ve Su İşleri Bakanlığı, Su Yönetimi Genel Müdürlüğü, Ankara (in Turkish). 